\title{
Renal Transplantation Survival Using
}

\section{ANN}

\author{
Manivasagam Rajendran ${ }^{\mathrm{a}, 1}$, and Vigneshwaran Perumal ${ }^{\mathrm{b}}$ \\ ${ }^{a}$ Associate Professor, Dept of EEE, K.Ramakrishnan College of Engineering, India \\ ${ }^{b}$ Assistant Professor, Dept of EEE, K.Ramakrishnan College of Engineering, India
}

\begin{abstract}
The aim of this paper is to study pre and post-transplant health records of kidney transplanted people and to find the importance of various factors in renal transplantation. Once the importance of these factors are quantified it can be used to predict the survival probability of the graft for given time. The possibility of using Artificial Neural Network (ANN) as a tool to predict the survival probability is discussed. Transplantation is the best option available to increase the life expectancy of the people who are suffering from end stage renal disease all over the world. The present system for organ procurement and management by the hospital and other reputed legal organizations are not sufficient to deal with the situation. The need of the hour is Systematic procedures for collection of organs, networking and sharing information about both pre transplantation and post transplantation levels. Application of scientific techniques and well proven methodologies of other domains like manufacturing and production can be utilized to improve the level of efficiency in organ transplants of our country.
\end{abstract}

Keywords: ANN, Survival Analysis, Renal Transplantation.

\section{Introduction}

Failure of kidney after transplantation in survival analysis, Cox's proportional. Hazards models given by Cox, D. R. (1972) have been traditionally used to discover attributes that are relevant to survival, and predict outcomes. Smith et al. (2000) transformed the output from Cox regression into survival estimation. However, the proportional hazards model is subject to a linear baseline. Cox regression makes two important assumptions about the hazard function: (1) Covariates affecting the hazard rate are independent, and (2) the ratio of risk in dying of two individuals is the same regardless of the time they have survived. De Laurentiis \& Ravdin (1994) suggested three situations in which artificial neural networks are better than Cox's regression model.

\section{Required Data}

Bertram et al (2010) analyzed 59,091 Chronic Kidney Disease (CKD) patients in USA using USRDBS (U.S Renal Data Base System). In their work, pretransplant, 7 days post transplant, 1 year post transplant, 5 year post transplant, patient's characteristics were observed. The factors considered by them are Recipient age, (yr), Recipient sex, Recipient race, Recipient Hispanic ethnicity, Recipient body mass index, $\mathrm{kg} / \mathrm{m}^{2}$, Primary cause of chronic kidney disease, Panel-reactive antibodies, Recipient HBSAg positive, Recipient HBV core antibody positive, Recipient HCV antibody positive,

\footnotetext{
${ }^{1}$ Manivasagam Rajendran: Asso. Professorr ,Dept of EEE, K.Ramakrishnan College of Engineering, India. Email: manivasagammn3@gmail.com.
} 
Recipient history of Diabetes, Hypertension, Tobacco use, Cancer, Limitations in activities of daily living, Pretransplant dialysis modality, Primary insurance coverage, Years on renal replacement therapy, Is it first kidney transplant, Donor age, Donor male sex, Donor race, Donor Hispanic ethnicity, Donor body mass index, Human leukocyte antigen mismatches, Cross-match positive, Cytomegalovirus $\mathrm{D}(+) / \mathrm{R}(-)$, Donor history of Smoking, Cancer, Diabetes, Hypertension, Donor HCV antibody positive, Right kidney used, Cold ischemia time, Donor terminal creatinine, Donor cocaine use, Donor non- intravenous drug use, Expanded criteria donor, Donation after cardiac death, Traumatic cause of donor death, eGFR in $\mathrm{mL} / \mathrm{min} / 1.73 \mathrm{~m} 2$ at discharge, Delayed graft function, Antibody induction at transplant, Functional status of the graft. In the absence of any central data base for collecting transplant data in India, it was not possible to collect all the 38 factors listed above. the factors that are usually recorded in Tamilnadu hospitals are found to be Recipient age, Primary cause of CKD, Discharge eGFR, Dialysis modality, HCV antibody, Donor age, Donor sex. Due to ethical issues and legal issues involved in giving data to third person's in the absence of any MoU only 22 samples were collected.

\section{3 . Artıficial Neural Network}

\section{ANN can be classified as}

\section{Feed Forward Net \\ II. Competitive Net \\ III. Recurrent Net.}

In this Paper, only feed forward network is used. So the abbreviation ANN refers to feed forward artificial neural network unless specified otherwise. ANN can be single layer or multilayer nets depending upon the number of hidden layers in the network. In a single layer ANN there will only input and output layer and no hidden layer.

Learning (Training): The process of setting weights for each link is called learning. Training an ANN is similar to teaching a child to identify things. Training is necessary for the child to identify things correctly. In this project supervised learning is used.

\section{Proposed Network}

In survival analysis time is an important factor. This project intends to predict survival probability of kidney transplanted patients for a given time i.e the probability of survival if a patient with the medical and physical parameters discussed in section 2 . is transplanted with donor characteristics listed in the same section at the end of $1^{\text {st }}$ year or $2^{\text {nd }}$ year or $3^{\text {rd }}$ year etc. So time should be included as one of the factor. It may be either dependent or independent factor. Two kinds of architecture are possible while including time.

1. Time taken as an input i.e. independent variable.

2. Dependent variable recorded at predefined time periods.

Architecture A: Instead of taking time as a continuous variable, the time horizon in which we are interested can be dived in to number of time periods. For example if we are interested in predicting survival probability for 5 year post transplant then we can divide that 5 year in to 10 periods of 6 months interval each. In this case 10 different networks are required each representing one time period. The dependent variable will be 
continuous between 0 and 1 . „0" means the graft didn"t survive that time period and, $1 "$ means the graft has survived that time period. In the case of missing patients who were unable to be tracked till that period the survival probability is calculated using Kaplan- Meir estimator given by the Eq.1.

$$
R(t)=\prod_{j: t_{j}<t}\left(1-1 / n_{j}\right)
$$

where

$\mathrm{R}(\mathrm{t})$ is the conditional survival probability of surviving past time ", $\mathrm{t}_{\mathrm{j}}$ " given that survival just prior to time $t_{j}$.

$>\quad t_{j}$ is the ordered failure times in ascending order.

$>\quad \mathrm{n}_{\mathrm{j}}$ is the number remaining at risk just prior to the $\mathrm{j}^{\text {th }}$ failure.

$>\quad \mathrm{I}_{1}, \mathrm{I}_{2} \ldots \mathrm{I}_{\mathrm{n}}$ are inputs (independent variables)

$\circ \quad$ is the output (dependent variable)

$>\quad$ W"s \& V"s are weights of the links connecting corresponding nodes.

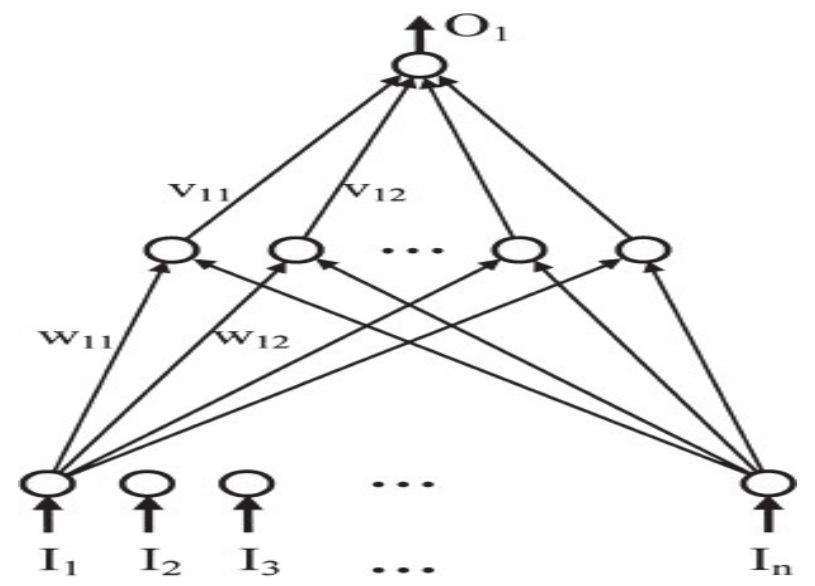

Figure 1. Architecture A

Table 1. Life expectancy -uncensored

\begin{tabular}{|c|c|c|c|c|c|c|c|c|}
\hline Month & $\begin{array}{l}0 \\
1 \\
0\end{array}$ & $\frac{1}{1}$ & $\frac{\infty}{\omega}$ & $\begin{array}{l}\stackrel{d}{d} \\
\text { a }\end{array}$ & $\begin{array}{l}0 \\
i n \\
n \\
n\end{array}$ & 站 & $\underset{\substack{1 \\
\text { In }}}{\text { m }}$ & \begin{tabular}{l}
$\infty$ \\
\multirow{1}{*}{} \\
$\dot{y}$
\end{tabular} \\
\hline period & P1 & P2 & P3 & P4 & P5 & P6 & P7 & P8 \\
\hline $\begin{array}{l}\text { Probability } \\
\text { of survival }\end{array}$ & 1 & 1 & 1 & 1 & 1 & 0 & 0 & 0 \\
\hline
\end{tabular}

Architecture B: In this architecture instead of training one network for each time period the dependent variable can be taken as an array in which each element of array gives the survival probability for each time period. For example if we are interested in predicting survival probability for 4 year post transplant then we can divide that 4 year in to 8 periods of 6 months interval each. The dependent variable will be an array of size 8 . The values of each element will be between 0 and 1 with „0" means the graft 
didn"t survive that time period and "1" means the graft has survived that time period. In the case of missing patients who were unable to be tracked till that period the survival probability is calculated using Kaplan-Meir estimator given by the Eq.1.

In this architecture one network is enough to predict survival probability for entire time we are concerned about. Only architecture B is used for further analysis for its simplicity. Let study period be 4 years and dividing this into time periods of 6 months the dependent variable i.e. life expectancy of a patient will be coded in to a binary form array of size 8 .

$>\quad \mathrm{I}_{1}, \mathrm{I}_{2} \ldots \mathrm{I}_{\mathrm{n}}$ are inputs (independent variables)

$>\mathrm{O}_{1}, \mathrm{O}_{2} \ldots \mathrm{O}_{\mathrm{n}}$ is the output with the suffix representing time period(dependent variable)

W"s \&V"s are weights of the links connecting corresponding nodes.

If the event occurs at $31^{\text {st }}$ month it will be coded as follows.

Due to some reasons if following up of patient is not possible after some time say 32 month, the dependent variable array will as one shown in Table 2. In this case Kaplan-Meir estimator is used to estimate the survival probability for period after right censoring.

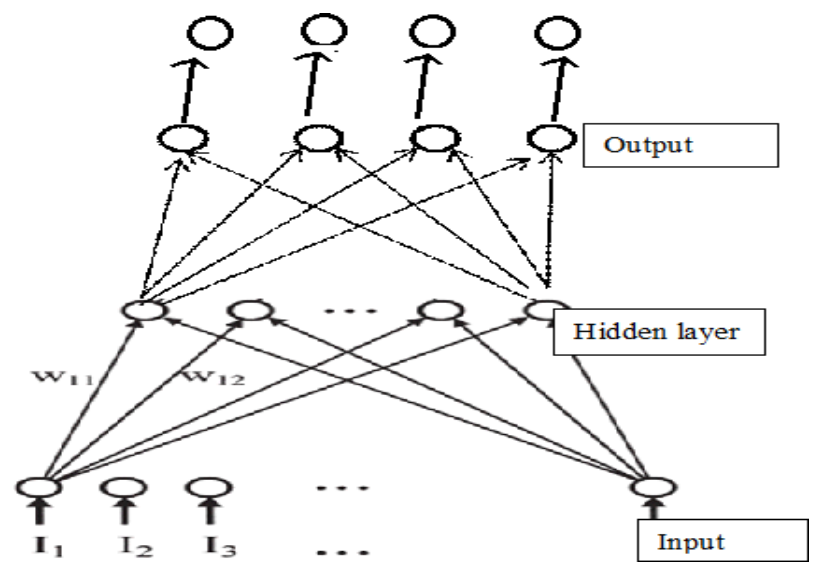

Figure 2. Architecture B

Table 2. Life expectancy -Right ...censored

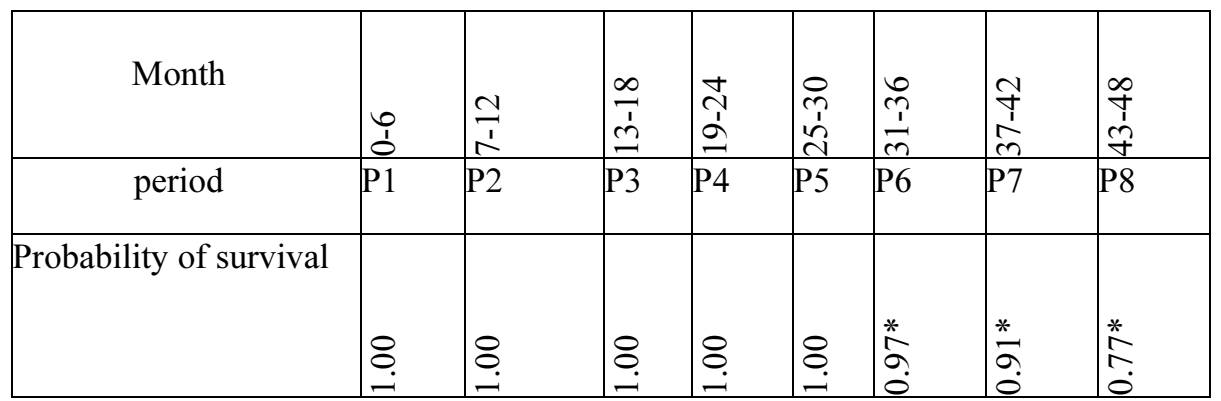




\section{Result}

Out of 22 samples, 2 were censored (not followed up). In five samples event has occurred (graft failed). The rest 15 are functioning. Feed Forward Multilayer perceptron model is used. Output nodes give the probability of survival for the corresponding period which is continous between 0 and 1 carried out. It shows that patient age (receptor) has highest importance followed. This is a preliminary result. More data has to be collected for practical application.

Let take that survival probability $\mathrm{P}(\mathrm{t})$ of kidney patients at time " Using the ANN model survival probability at time ,t" can be predicted. Probability of not surviving can be taken as risk $\mathrm{R}(\mathrm{t})$.

$$
R(t)=1-P(t)
$$

This risk $\mathrm{R}(\mathrm{t})$ is to be minimized. This can be constructed as an assignment problem.

$$
\text { Minimize } \quad \sum_{j=1}^{n} \sum_{i=1}^{n} a_{i j} R(t)_{i j}
$$

Subjected to the constraints 1 .

$$
\sum_{j=1}^{n} a_{i j}=1 \quad \forall i
$$

where

$a_{i j}$ is a binary variable. $i, j$ gives kidney and patient identity respectively. $R(t)_{i j}$ gives the predicted risk involved transplanting the kidney of $\mathrm{i}^{\text {th }}$ donor to $\mathrm{j}^{\text {th }}$ patient.Using the concept of paired kidney exchange, list exchange transplants about to be performed in a short period of time say within a week can be pooled and pairing can be done among those patients and donors such that the survival probability is maximized for every patient.

\section{References}

[1] Bertram, L.K., Ajay, K.I., Jon,J.S., Melissa A.S., Yipeng, and Eric D.W., "A simple tool to predict outcomes after kidney transplant”., American Journal of Kidney Disease. Vol. 56, 2010, pp. 947-960.

[2] Brier, M.V., Ray,P.C., Klein,J.B.," Prediction of delayed renal allograft function using an artificial neural network"., Nephrol Dial Transplant. ,2003, pp2655-2659.

[3] Chih-Lin, C., Street,W.N., Wolberg,W.H., "Application of Artificial Neural Network-Based Survival Analysis on Two Breast Cancer Datasets”., AMIA Symposium Proceedings., 2007, pp130-134.

[4] Cox, D.R.,"Regression Models and Life- Tables"Journal of the Royal Statistical Society. Series B (Methodological)., Vol. 34 No. 2, 1972, pp. 187-220.

[5] De Laurentiis, M.. and Ravdin, P. M. "A technique for using neural network analysis to perform survival analysis of censored data"., Cancer Letters, Vol.77, 1994,pp.127- 138.

[6] Goldfarb-Rumyantzev, A.S., Scandling, J.D., Pappas, L., Smout, R.J., and Horn, S. "Prediction of 3-yr cadaveric graft survival based on pre-transplant variables in a large national dataset".Clinical Transplant., Vol. 17, 2003, pp. 485-497.

[7] R. Manivasagam, P. Parthasarathy, and R. Anbumozhi, "Robust analysis of T-S fuzzy controller for nonlinear system using H-infinity," Advances in Intelligent Systems and Computing, vol. 949, pp. 643-651, 2019. Available at: https://doi.org/10.1007/978-981-13-8196-6_56.

[8] Gumber, M.R., Goplani, K.R., Shah, P.R., and Patel, H.V. "Study on deceased donor organ transplantation". Indian Journal of Nephrology, Vol. 21, 2011, Issue 3, pp. 182-186

[9] Hariharan, S., McBride, M.A., Cherikh, W.S., Tolleris, C.B., Bresnahan, B.A., and Johnson, C.P.,"Post-transplant renal function in the first year predicts long-term kidney transplant survival", Kidney International. Vol.62, 2002, pp. 311-318

[10] cadaver and living related renal transplant recipients", Nephrol Dial Transplant, Vol 17,2002, pp. 2204-2211

[11]Long, J.S., "Regression models for categorical and limited dependent variables"., Sage publications, Thousand Oaks, London.

[12] Machnicki, G., Pinsky, B., and Takemoto, S., "Predictive ability of pretransplant comorbidities to predict long-term graft loss and death"., American Journal of Transplant., Vol. 9, 2009, pp. 494-50. 
[13]R. Manivasagam and V. Dharmalingam, "Power quality problem mitigation by unified power quality conditioner: An adaptive hysteresis control technique," International Journal of Power Electronics, vol. 6, pp. 403-425, 2014. Available at: https://doi.org/10.1504/ijpelec.2014.067442.

[14] Ojo, A.O., Hanson, J.A., Wolfe, R.A., Leichtman, A.B., Agodoa, L.Y., and Port, F.K., "Long-term survival in renal transplant recipients with graft function". Kidney International., Vol. 57, 2000, pp.307-313. 